Article

\title{
On Some Generalized Fractional Integral Inequalities for $p$-Convex Functions
}

\author{
Seren Salaş, Yeter Erdaş, Tekin Toplu and Erhan Set *๑
}

Department of Mathematics, Faculty of Science and Arts, Ordu University, 52200 Ordu, Turkey; serensalas@gmail.com (S.S.); yeterrerdass@gmail.com (Y.E.); tekintoplu@gmail.com (T.T.)

* Correspondence: erhanset@yahoo.com

Received: 15 April 2019; Accepted: 8 May 2019; Published: 20 May 2019

check for updates

\begin{abstract}
In this paper, firstly we have established a new generalization of Hermite-Hadamard inequality via $p$-convex function and fractional integral operators which generalize the Riemann-Liouville fractional integral operators introduced by Raina, Lun and Agarwal. Secondly, we proved a new identity involving this generalized fractional integral operators. Then, by using this identity, a new generalization of Hermite-Hadamard type inequalities for fractional integral are obtained.
\end{abstract}

Keywords: $p$-convex function; Hermite-Hadamard inequality; fractional integral operator

MSC: 26A33, 26D10, 26D15

\section{Introduction}

In mathematical literature (see, [1] and references therein ), the Hermite-Hadamard inequality, named after Charles Hermite (1822-1901) and Jacques Hadamard (1865-1963) and sometimes also called Hadamard's inequality, states that if a function $f:[a, b] \longrightarrow \mathbb{R}$ is convex, then the following chain of inequalities holds:

$$
f\left(\frac{a+b}{2}\right) \leq \frac{1}{b-a} \int_{a}^{b} f(x) d x \leq \frac{f(a)+f(b)}{2} .
$$

The inequality (1) is one of the most famous result for convex functions. A number of paper have been written on this inequality providing new proofs note worthy extensions, generalizations, refinements and new inequalities connected with the Hermite-Hadamard inequality. Since then, the inequality (1) has attracted many mathematicians attention. Especially, in the last three decades, numerous generalizations, variants and extensions of this inequality have been presented (see, e.g., [1-7]) and the references cited therein.

In [8], Zhang and Wan gave definition of $p$-convex function as follows.

Definition 1. Let I be a p-convex set. A function $f: I \longrightarrow \mathbb{R}$ is said to be a $p$-convex function or belongs to class $P C(I)$, if

$$
f\left(\left[t x^{p}+(1-t) y^{p}\right]^{\frac{1}{p}}\right) \leq t f(x)+(1-t) f(y)
$$

for all $x, y \in I$ and $t \in[0,1]$

Remark 1 ([8]). An interval $I$ is said to be a $p$-convex set if $\left[t x^{p}+(1-t) y^{p}\right]^{\frac{1}{p}} \in I$ for all $x, y \in I$ and $t \in[0,1]$, where $p=2 k+1$ or $p=\frac{n}{m}, n=2 r+1, m=2 s+1$ and $k, r, s \in \mathbb{N}$. 
Definition 2 ([8]). If $I \subset(0, \infty)$ be a real interval and $p \in \mathbb{R} \backslash 0$, then $\left[t x^{p}+(1-t) y^{p}\right]^{\frac{1}{p}} \in I$ for all $x, y \in I$ and $t \in[0,1]$.

According to Remark (1), a different version of definition of $p$-convex function was given by İşcan as below.

Definition 3 ([9]). Let $I \subset(0, \infty)$ be a real interval and $p \in \mathbb{R} \backslash 0$. A function $f: I \longrightarrow \mathbb{R}$ is said to be a p-convex function, if

$$
f\left(\left[t x^{p}+(1-t) y^{p}\right]^{\frac{1}{p}}\right) \leq t f(x)+(1-t) f(y)
$$

for all $x, y \in I$ and $t \in[0,1]$. If the inequality is reversed, then $f$ is said to be $p$-concave.

According to definition above, it can easily be seen that for $p=1$ and $p=-1, p$-convexity reduces to ordinary convexity and harmonically convexity [10] of functions defined on $I \subset(0, \infty)$, respectively.

The Hermite-Hadamard inequality for $p$-convex functions is as the following (see [11,12])

Theorem 1. $f: I \longrightarrow \mathbb{R}$ be a p-convex function and $a, b \in I$ with $a<b$. If $f \in L[a, b]$, then we have

$$
f\left(\left[\frac{a^{p}+b^{p}}{2}\right]^{\frac{1}{p}}\right) \leq \frac{p}{b^{p}-a^{p}} \int_{a}^{b} \frac{f(x)}{x^{1-p}} d x \leq \frac{f(a)+f(b)}{2} .
$$

For some result related to $p$-convex functions and its generalizations, we refer the reader to see now $[8,9,11-18]$.

We recall following special functions (see $[19,20]$ )

1. The Gamma Function :

The Gamma $\Gamma$ functions are defined by

$$
\Gamma(\alpha)=\int_{0}^{\infty} e^{-t} t^{\alpha} d t, \alpha>0 .
$$

The gamma function is a natural extension of the factorial from integers $n$ to real numbers $\alpha$.

2. The Beta Function:

$$
\beta(x, y)=\frac{\Gamma(x) \Gamma(y)}{\Gamma(x+y)}=\int_{0}^{1} t^{x-1}(1-y)^{y-1} d t \quad x, y>0
$$

3. The Hypergeometric Function:

$$
{ }_{2} F_{1}(a, b ; c, z)=\frac{1}{\beta(b, c-b)} \int_{0}^{1} t^{b-1}(1-t)^{c-b-1}(1-z t)^{-a} d t, c>b>0,|z|<1
$$

Definition 4. Let $[a, b]$ be a finite interval on the real axis $\mathbb{R}$ and $f \in[a, b]$. The right-hand side and left-hand side Riemann-Liouville fractional integrals $J_{a^{+}}^{\alpha} f$ and $J_{b^{-}}^{\alpha} f$ of order $\alpha>0$ are defined by

$$
\begin{aligned}
& J_{a^{+}}^{\alpha} f(x)=\frac{1}{\Gamma(\alpha)} \int_{a}^{x}(x-t)^{\alpha-1} f(t) d t, x>a \\
& J_{b^{-}}^{\alpha} f(x)=\frac{1}{\Gamma(\alpha)} \int_{x}^{b}(t-x)^{\alpha-1} f(t) d t, x<b
\end{aligned}
$$

respectively.

For more details and properties concerning with this fractional integral operators defination (4), we refer the reader, for example, to [2,19,21-24] and the references therein. In [4] Sarikaya et al. proved the following inequality for fractional integrals. 
Theorem 2. Let $f:[a, b] \longrightarrow \mathbb{R}$ be a positive function with $0 \leq a<b$ and $f \in L_{1}[a, b]$. If $f$ is convex function on $[a, b]$, then the following inequality for fractional integrals holds:

$$
f\left(\frac{a+b}{2}\right) \leq \frac{\Gamma(\alpha+1)}{2(b-a)^{\alpha}}\left[J_{a^{+}}^{\alpha} f(b)+J_{b^{-}}^{\alpha} f(a)\right] \leq \frac{f(a)+f(b)}{2}
$$

with $\alpha>0$.

Raina [25] introduced a class of functions defined formally by

$$
\mathcal{F}_{\rho, \lambda}^{\sigma}(x)=\mathcal{F}_{\rho, \lambda}^{\sigma(0), \sigma(1), \ldots}(x)=\sum_{k=0}^{\infty} \frac{\sigma(k)}{\Gamma(\rho k+\lambda)} x^{k}\left(\rho, \lambda \in \mathbb{R}^{+} ;|x|<\mathbb{R}\right)
$$

where the coefficients $\sigma(k) \in \mathbb{R}^{+}\left(k \in \mathbb{N}_{0}\right)$ form a bounded sequence. With the help of (3) Raina [25] and Agarwal et al. [26] defined, respectively, the following left-side and right-sided fractional integral operators:

$$
\begin{aligned}
& \left(j_{\rho, \lambda, a+; \omega}^{\sigma} \varphi\right)(x)=\int_{a}^{x}(x-t)^{\lambda-1} \mathcal{F}_{\rho, \lambda}^{\sigma}\left[\omega(x-t)^{\rho}\right] \varphi(t) d t(x>a) \\
& \left(j_{\rho, \lambda, b-; w}^{\sigma} \varphi\right)(x)=\int_{x}^{b}(t-x)^{\lambda-1} \mathcal{F}_{\rho, \lambda}^{\sigma}\left[\omega(t-x)^{\rho}\right] \varphi(t) d t(x<b),
\end{aligned}
$$

where $\lambda, \rho \in \mathbb{R}^{+}, \omega \in \mathbb{R}$ and $\varphi(t)$ is a function such that the integrals on right sides exits.

It is easy to verify that $\left(J_{\rho, \lambda, a+; w}^{\sigma} \varphi\right)(x)$ and $\left(J_{\rho, \lambda, b-; w}^{\sigma} \varphi\right)(x)$ are bounded integral operators on $L(a, b)$, if

$$
\mathfrak{M}:=\mathcal{F}_{\rho, \lambda+1}^{\sigma}\left[\omega(b-a)^{\rho}\right]<\infty .
$$

In fact,

$$
\left\|j_{\rho, \lambda, a+; w}^{\sigma} \varphi\right\|_{1} \leq \mathfrak{M}(b-a)^{\lambda}\|\varphi\|_{1} \quad(\varphi(t) \in L(a, b))
$$

and

$$
\left\|\int_{\rho, \lambda, b-; w}^{\sigma} \varphi\right\|_{1} \leq \mathfrak{M}(b-a)^{\lambda}\|\varphi\|_{1}(\varphi(t) \in L(a, b)) .
$$

Here, many useful fractional integral operators can be obtained by specializing the function $\mathcal{F}_{\rho, \lambda}^{\sigma}(x)$. For instance, the classical Riemann-Liouville fractional integrals $J_{a^{+}}^{\alpha}$ and $J_{b^{-}}^{\alpha}$ of order $\alpha$ follow easily by setting $\lambda=\alpha, \sigma(0)=1$ and $\omega=0$ in (4) and (5). Sarkkaya and Yaldız [7] proved Hermite-Hadamard type inequality involving the fractional integral operators (4) and (5) asserted by the following theorem.

Theorem 3. Let $\lambda \in \mathbb{R}^{+}$and $\varphi:[a, b] \rightarrow \mathbb{R}(a<b)$ be a convex function on $[a, b]$. Then

$$
\begin{aligned}
\varphi\left(\frac{a+b}{2}\right) & \left.\leq \frac{1}{2(b-a)^{\lambda} \mathcal{F}_{\rho, \lambda+1}^{\sigma}\left[w(b-a)^{\rho}\right]}[]_{\rho, \lambda, a+; w}^{\sigma} f(b)+j_{\rho, \lambda, b-; w}^{\sigma} f(a)\right] \\
& \leq \frac{\varphi(a)+\varphi(b)}{2}
\end{aligned}
$$

The aim of this paper is to establish new Hermite-Hadamard type inequalities for $p$-convex functions in terms of generalized fractional integral operators. 


\section{Main Results}

In the section, using generalized fractional integral operators, we start with stating and proving the fractional integral counter of Theorem 4, Lemma 1 and Theorem 5 then some other refinements will follow. We begin by the following theorem:

Theorem 4. Let $\lambda \in \mathbb{R}^{+}, f: I \subseteq \mathbb{R} \longrightarrow \mathbb{R}$ be a p-convex function and $a, b \in I$ with $a<b, p>0$. If $f \in L[a, b]$, then we have

$$
\begin{aligned}
& f\left(\left[\frac{a^{p}+b^{p}}{2}\right]^{1 / p}\right) \\
& \leq \frac{1}{2 \mathcal{F}_{\rho, \lambda+1}^{\sigma}\left[w\left(b^{p}-a^{p}\right)^{\rho}\right]\left(b^{p}-a^{p}\right)^{\lambda}}\left[J_{\rho, \lambda, a^{p}+; w}^{\sigma} f \circ g\left(b^{p}\right)+J_{\rho, \lambda, b^{p}-; w}^{\sigma} f \circ g\left(a^{p}\right)\right] \\
& \leq \frac{f(a)+f(b)}{2}
\end{aligned}
$$

where $g(x)=x^{\frac{1}{p}}$.

Proof. Since $f$ is $p$-convex function on $[a, b]$, we have for all $x, y \in[a, b]$

$$
f\left(\left[\frac{x^{p}+y^{p}}{2}\right]^{\frac{1}{p}}\right) \leq \frac{f(x)+f(y)}{2} .
$$

Choosing $x=\left[t a^{p}+(1-t) b^{p}\right]^{\frac{1}{p}}$ and $y=\left[(1-t) a^{p}+t b^{p}\right]^{\frac{1}{p}}$, then we get

$$
2 f\left(\left[\frac{a^{p}+b^{p}}{2}\right]^{\frac{1}{p}}\right) \leq f\left(\left[t a^{p}+(1-t) b^{p}\right]^{\frac{1}{p}}\right)+f\left(\left[(1-t) a^{p}+t b^{p}\right]^{\frac{1}{p}}\right) .
$$

Multiplying both sides of the inequality (8) by $t^{\lambda-1} \mathcal{F}_{\rho, \lambda}^{\sigma}\left[w\left(b^{p}-a^{p}\right)^{\rho} t^{\rho}\right]$ and then integrating the resulting inequality with respect to $t$ over $[0,1]$, then we obtain

$$
\begin{aligned}
& 2 \mathcal{F}_{\rho, \lambda+1}^{\sigma}\left[w\left(b^{p}-a^{p}\right)^{\rho}\right] f\left(\left[\frac{a^{p}+b^{p}}{2}\right]\right)^{\frac{1}{p}} \\
\leq & \int_{0}^{1} t^{\lambda-1} \mathcal{F}_{\rho, \lambda}^{\sigma}\left[w\left(b^{p}-a^{p}\right)^{\rho} t^{\rho}\right] f\left(\left[t a^{p}+(1-t) b^{p}\right]^{\frac{1}{p}}\right) d t \\
+ & \int_{0}^{1} t^{\lambda-1} \mathcal{F}_{\rho, \lambda}^{\sigma}\left[w\left(b^{p}-a^{p}\right)^{\rho} t^{\rho}\right] f\left(\left[(1-t) a^{p}+t b^{p}\right]^{\frac{1}{p}}\right) d t
\end{aligned}
$$




$$
\begin{aligned}
& =\int_{0}^{1} t^{\lambda-1} \sum_{k=0}^{\infty} \frac{\sigma(k) w^{k}\left(b^{p}-a^{p}\right)^{\rho k} t^{\rho k}}{\Gamma(\rho k+\lambda)} f\left(\left[t a^{p}+(1-t) b^{p}\right]^{\frac{1}{p}}\right) d t \\
& +\int_{0}^{1} t^{\lambda-1} \sum_{k=0}^{\infty} \frac{\sigma(k) w^{k}\left(b^{p}-a^{p}\right)^{\rho k} t^{\rho k}}{\Gamma(\rho k+\lambda)} f\left(\left[(1-t) a^{p}+t b^{p}\right]^{\frac{1}{p}}\right) d t \\
& =\int_{0}^{1} \sum_{k=0}^{\infty} \frac{\sigma(k) w^{k}}{\Gamma(\rho k+\lambda)} t^{\rho k+\lambda-1} f\left(\left[t a^{p}+(1-t) b^{p}\right]^{\frac{1}{p}}\right)\left(b^{p}-a^{p}\right)^{\rho k} d t \\
& +\int_{0}^{1} \sum_{k=0}^{\infty} \frac{\sigma(k) w^{k}}{\Gamma(\rho k+\lambda)} t^{\rho k+\lambda-1} f\left(\left[(1-t) a^{p}+t b^{p}\right]^{\frac{1}{p}}\right)\left(b^{p}-a^{p}\right)^{\rho k} d t \\
& =\int_{a^{p}}^{b^{p}} \sum_{k=0}^{\infty} \frac{\sigma(k) w^{k}}{\Gamma(\rho k+\lambda)} \frac{\left(b^{p}-x\right)^{\rho k}\left(b^{p}-x\right)^{\lambda-1}}{\left(b^{p}-a^{p}\right)^{\rho k}\left(b^{p}-a^{p}\right)^{\lambda-1}}\left(b^{p}-a^{p}\right)^{\rho k} f\left(x^{\frac{1}{p}}\right) \frac{d x}{b^{p}-a^{p}} \\
& +\int_{a^{p}}^{b^{p}} \sum_{k=0}^{\infty} \frac{\sigma(k) w^{k}}{\Gamma(\rho k+\lambda)} \frac{\left(x-a^{p}\right)^{\rho k}\left(x-a^{p}\right)^{\lambda-1}}{\left(b^{p}-a^{p}\right)^{\rho k}\left(b^{p}-a^{p}\right)^{\lambda-1}}\left(b^{p}-a^{p}\right)^{\rho k} f\left(x^{\frac{1}{p}}\right) \frac{d x}{b^{p}-a^{p}} \\
& =\frac{1}{\left(b^{p}-a^{p}\right)^{\lambda}} \int_{a^{p}}^{b^{p}} \sum_{k=0}^{\infty} \frac{\sigma(k) w^{k}\left(b^{p}-x\right)^{\rho k}}{\Gamma(\rho k+\lambda)}\left(b^{p}-x\right)^{\lambda-1} f\left(x^{\frac{1}{p}}\right) d x \\
& +\frac{1}{\left(b^{p}-a^{p}\right)^{\lambda}} \int_{a^{p}}^{b^{p}} \sum_{k=0}^{\infty} \frac{\sigma(k) w^{k}\left(x-a^{p}\right)^{\rho k}}{\Gamma(\rho k+\lambda)}\left(x-a^{p}\right)^{\lambda-1} f\left(x^{\frac{1}{p}}\right) d x \\
& =\frac{1}{\left(b^{p}-a^{p}\right)^{\lambda}}\left(J_{\rho, \lambda, a^{p}+; w}^{\sigma} f \circ g\right)\left(b^{p}\right) \\
& +\frac{1}{\left(b^{p}-a^{p}\right)^{\lambda}}\left(J_{\rho, \lambda, b^{p}-; w}^{\sigma} f \circ g\right)\left(a^{p}\right) \text {. }
\end{aligned}
$$

Thus we have

$$
2 \mathcal{F}_{\rho, \lambda+1}^{\sigma}\left[w\left(b^{p}-a^{p}\right)^{\sigma}\right] f\left(\left[\frac{a^{p}+b^{p}}{2}\right]^{1 / p}\right) \leq \frac{1}{\left(b^{p}-a^{p}\right)^{\lambda}}\left[\left(J_{\rho, \lambda, b^{p}-; w}^{\sigma} f \circ g\right)\left(a^{p}\right)+\left(J_{\rho, \lambda, a^{p}+; w}^{\sigma} f \circ g\right)\left(b^{p}\right)\right]
$$

which completes the proof of the first inequality.

Now we will prove the right-side of the inequality in (8). Using the $p$-convexity of $f$

$$
f\left(\left[t a^{p}+(1-t) b^{p}\right]^{\frac{1}{p}}\right) \leq t f(a)+(1-t) f(b)
$$

and

$$
f\left(\left[(1-t) a^{p}+t b^{p}\right]^{\frac{1}{p}}\right) \leq(1-t) f(a)+t f(b) .
$$

By adding these inequalities, then we have

$$
f\left(\left[t a^{p}+(1-t) b^{p}\right]^{\frac{1}{p}}\right)+f\left(\left[(1-t) a^{p}+t b^{p}\right]^{\frac{1}{p}}\right) \leq f(a)+f(b) .
$$

Multiplying both sides of inequality by $t^{\lambda-1} \mathcal{F}_{\rho, \lambda}^{\sigma}\left[w\left(b^{p}-a^{p}\right)^{\rho} t^{\rho}\right]$, and then integrating the resulting inequality with respest to $t$ over $[0,1]$, we obtain

$$
\frac{1}{\left(b^{p}-a^{p}\right)^{\lambda}}\left[\left(J_{\rho, \lambda, a^{p}+; w}^{\sigma} f \circ g\right)\left(b^{p}\right)+\left(J_{\rho, \lambda, b^{p}-; w}^{\sigma}\right) f \circ g\left(a^{p}\right)\right] \leq 2 \mathcal{F}_{\rho, \lambda+1}^{\sigma}\left[w\left(b^{p}-a^{p}\right)^{\rho}\right] \frac{f(a)+f(b)}{2} .
$$

This completes the proof. 
Lemma 1. Let $\lambda \in \mathbb{R}^{+} \varphi: I \subseteq \mathbb{R}^{+} \rightarrow \mathbb{R}$ be a differentiable mapping on $I^{\circ} a, b \in I$ with $a<b, p>0$. If $f^{\prime} \in L[a, b]$, then the following equality the generalized fractional integrals holds:

$$
\begin{aligned}
& \frac{f(a)+f(b)}{2}-\frac{1}{2 \mathcal{F}_{\rho, \lambda+1}^{\sigma}\left[\omega\left(b^{p}-a^{p}\right)^{\rho}\right]\left(b^{p}-a^{p}\right)^{\lambda}}\left[\left(\int_{\rho, \lambda, a^{p}+; \omega}^{\sigma} f \circ g\right)\left(b^{p}\right)+\left(j_{\rho, \lambda, b^{p}-; \omega}^{\sigma} f \circ g\right)\left(a^{p}\right)\right] \\
& =\frac{\left(b^{p}-a^{p}\right)}{2 p \mathcal{F}_{\rho, \lambda+1}^{\sigma}\left[\omega\left(b^{p}-a^{p}\right)^{\rho}\right]}\left[\begin{array}{l}
\int_{0}^{1}(1-t)^{\lambda} \mathcal{F}_{\rho, \lambda+1}^{\sigma}\left[\omega\left(b^{p}-a^{p}\right)^{\rho}(1-t)^{\rho}\right] \\
\left(t a^{p}+(1-t) b^{p}\right)^{\frac{1}{p}-1} f^{\prime}\left(\left(t a^{p}+(1-t) b^{p}\right)^{\frac{1}{p}}\right) d t \\
+\int_{0}^{1} t^{\lambda} \mathcal{F}_{\rho, \lambda+1}^{\sigma}\left[\omega\left(b^{p}-a^{p}\right)^{\rho} t^{\rho}\right] \\
\left(t a^{p}+(1-t) b^{p}\right)^{\frac{1}{p}-1} f^{\prime}\left(\left(t a^{p}+(1-t) b^{p}\right)^{\frac{1}{p}}\right) d t
\end{array}\right]
\end{aligned}
$$

where $g(x)=x^{\frac{1}{p}}$.

Proof. Here, we apply integration by parts in integrals of right part of (9), then

$$
\begin{aligned}
& \int_{0}^{1}(1-t)^{\lambda} \mathcal{F}_{\rho, \lambda+1}^{\sigma}\left[\omega\left(b^{p}-a^{p}\right)^{\rho}(1-t)^{\rho}\right]\left(t a^{p}+(1-t) b^{p}\right)^{\frac{1}{p}-1} f^{\prime}\left(\left(t a^{p}+(1-t) b^{p}\right)^{\frac{1}{p}}\right) d t \\
& +\int_{0}^{1} t^{\lambda} \mathcal{F}_{\rho, \lambda+1}^{\sigma}\left[\omega\left(b^{p}-a^{p}\right)^{\rho} t^{\rho}\right]\left(t a^{p}+(1-t) b^{p}\right)^{\frac{1}{p}-1} f^{\prime}\left(\left(t a^{p}+(1-t) b^{p}\right)^{\frac{1}{p}}\right) d t \\
& =\left.(1-t)^{\lambda} \mathcal{F}_{\rho, \lambda+1}^{\sigma}\left[\omega\left(b^{p}-a^{p}\right)^{\rho}(1-t)^{\rho}\right] \frac{p}{\left(a^{p}-b^{p}\right)} f\left(\left(t a^{p}+(1-t) b^{p}\right)^{\frac{1}{p}}\right)\right|_{0} ^{1} \\
& -\int_{0}^{1}(1-t)^{\lambda-1} \mathcal{F}_{\rho, \lambda}^{\sigma}\left[\omega\left(b^{p}-a^{p}\right)^{\rho}(1-t)^{\rho}\right] \frac{p}{\left(a^{p}-b^{p}\right)} f\left(\left(t a^{p}+(1-t) b^{p}\right)^{\frac{1}{p}}\right) d t \\
& +\left.t^{\lambda} \mathcal{F}_{\rho, \lambda+1}^{\sigma}\left[\omega\left(b^{p}-a^{p}\right)^{\rho} t^{\rho}\right] \frac{p}{\left(a^{p}-b^{p}\right)} f\left(\left(t a^{p}+(1-t) b^{p}\right)^{\frac{1}{p}}\right)\right|_{0} ^{1} \\
& -\int_{0}^{1} t^{\lambda-1} \mathcal{F}_{\rho, \lambda}^{\sigma}\left[\omega\left(b^{p}-a^{p}\right)^{\rho} t^{\rho}\right] \frac{p}{\left(a^{p}-b^{p}\right)} f\left(\left(t a^{p}+(1-t) b^{p}\right)^{\frac{1}{p}}\right) d t \\
& =\frac{p}{\left(a^{p}-b^{p}\right)} \mathcal{F}_{\rho, \lambda+1}^{\sigma}\left[\omega\left(b^{p}-a^{p}\right)^{\rho} f(b)-\frac{p}{\left(a^{p}-b^{p}\right)} \int_{0}^{1}(1-t)^{\lambda-1} \mathcal{F}_{\rho, \lambda}^{\sigma}\left[\omega\left(b^{p}-a^{p}\right)^{\rho}(1-t)^{\rho}\right] f\left(\left(t a^{p}+(1-t) b^{p}\right)^{\frac{1}{p}}\right) d t\right. \\
& +\frac{p}{\left(a^{p}-b^{p}\right)} \mathcal{F}_{\rho, \lambda+1}^{\sigma}\left[\omega\left(b^{p}-a^{p}\right)^{\rho} f(a)-\frac{p}{\left(a^{p}-b^{p}\right)} \int_{0}^{1} t^{\lambda-1} \mathcal{F}_{\rho, \lambda}^{\sigma}\left[\omega\left(b^{p}-a^{p}\right)^{\rho} t t^{\rho}\right] f\left(\left(t a^{p}+(1-t) b^{p}\right)^{\frac{1}{p}}\right) d t\right. \\
& =\frac{p}{\left(a^{p}-b^{p}\right)} \mathcal{F}_{\rho, \lambda+1}^{\sigma}\left[\omega\left(b^{p}-a^{p}\right)^{\rho}\right][f(a)+f(b)]-\frac{p}{\left(b^{p}-a^{p}\right)^{\lambda+1}}\left[J_{\rho, \lambda, a^{p}+; \omega}^{\sigma} f o g\left(b^{p}\right)+J_{\rho, \lambda}^{\sigma}, b^{p}-; \omega f o g\left(a^{p}\right)\right] .
\end{aligned}
$$

Multiplying both sides (10), by $\frac{\left(a^{p}-b^{p}\right)}{2 p \mathcal{F}_{\rho, \lambda+1}^{\sigma}\left[\left(b^{p}-a^{p}\right)^{\rho}\right]}$ we have (9). Thus we accomplish proof of this lemma.

Theorem 5. Let $\lambda \in \mathbb{R}^{+} f: I \subseteq \mathbb{R}^{+} \rightarrow \mathbb{R}$ be a differentiable mapping on $I^{\circ} a, b \in I^{\circ}$ with $a<b$ and $p>0$. If $\left|f^{\prime}\right|$ is $p$-convex on $[a, b]$, then the following inequality for generalized fractional integrals operators holds:

$$
\begin{aligned}
& \left|\frac{f(a)+f(b)}{2}-\frac{1}{2\left(b^{p}-a^{p}\right)^{\lambda} \mathcal{F}_{\rho, \lambda+1}^{\sigma}\left[w\left(b^{p}-a^{p}\right)^{\rho}\right]}\left[\left(J_{\rho, \lambda, a^{p}+; w}^{\sigma} f \circ g\right)\left(b^{p}\right)+\left(J_{\rho, \lambda, b^{p}-; w}^{\sigma} f o g\right)\left(a^{p}\right)\right]\right| \\
\leq & \left(\mathcal{F}_{\rho, \lambda+1}^{\sigma_{1}}\left[|w|\left(b^{p}-a^{p}\right)^{\rho}\right]+\mathcal{F}_{\rho, \lambda+1}^{\sigma_{3}}\left[|w|\left(b^{p}-a^{p}\right)^{\rho}\right]\right)\left|f^{\prime}(a)\right|+\left(\mathcal{F}_{\rho, \lambda+1}^{\sigma_{2}}\left[w\left(b^{p}-a^{p}\right)^{\rho}\right]+\mathcal{F}_{\rho, \lambda+1}^{\sigma_{4}}\left[w\left(b^{p}-a^{p}\right)^{\rho}\right]\right)\left|f^{\prime}(b)\right|
\end{aligned}
$$

where

$$
\begin{aligned}
& \sigma_{1}=\sigma(k) b^{1-p} \frac{2}{(\rho k+\lambda+1)(\rho k+\lambda+2)}\left[{ }_{2} \mathcal{F}_{1}\left(1-\frac{1}{p^{\prime}}, 2 ; \rho k+\lambda+3,1-\frac{a^{p}}{b^{p}}\right)\right] \\
& \sigma_{2}=\sigma(k) b^{1-p} \frac{1}{\rho k+\lambda+2}\left[{ }_{2} \mathcal{F}_{1}\left(1-\frac{1}{p}, 1 ; \rho k+\lambda+3,1-\frac{a^{p}}{b^{p}}\right)\right] \\
& \sigma_{3}=\sigma(k) b^{1-p} \frac{1}{\rho k+\lambda+1}\left[{ }_{2} \mathcal{F}_{1}\left(1-\frac{1}{p^{\prime}} \rho k+\lambda+2 ; \rho k+\lambda+3,1-\frac{a^{p}}{b^{p}}\right)\right] \\
& \sigma_{4}=\sigma(k) b^{1-p} \frac{2}{(\rho k+\lambda+1)(\rho k+\lambda+2)}\left[{ }_{2} \mathcal{F}_{1}\left(1-\frac{1}{p^{\prime}}, \rho k+\lambda+1 ; \rho k+\lambda+3,1-\frac{a^{p}}{b^{p}}\right)\right] .
\end{aligned}
$$


Proof. Using Lemma 1 and $p$-convexity of $\left|f^{\prime}\right|$ we have,

$$
\begin{aligned}
& \left|\frac{f(a)+f(b)}{2}-\frac{1}{2\left(b^{p}-a^{p}\right)^{\lambda} \mathcal{F}_{\rho, \lambda+1}^{\sigma}\left[w\left(b^{p}-a^{p}\right)^{\rho}\right]}\left[\left(J_{\rho, \lambda, a^{p}+; w}^{\sigma} f o g\right)\left(b^{p}\right)+\left(J_{\rho, \lambda, b^{p}-; w}^{\sigma} f o g\right)\left(a^{p}\right)\right]\right| \\
= & \mid \frac{b^{p}-a^{p}}{2 p \mathcal{F}_{\rho, \lambda+1}^{\sigma}\left[w\left(b^{p}-a^{p}\right)^{\rho}\right]}\left[\int_{0}^{1}(1-t)^{\lambda} \mathcal{F}_{\rho, \lambda+1}^{\sigma}\left[w\left(b^{p}-a^{p}\right)^{\rho}(1-t)^{\rho}\right]\right. \\
& \times\left(t a^{p}+(1-t) b^{p}\right)^{\frac{1}{p}-1} f^{\prime}\left(t a^{p}+(1-t) b^{p}\right)^{\frac{1}{p}} d t \\
& +\int_{0}^{1} t^{\lambda} \mathcal{F}_{\rho, \lambda+1}^{\sigma}\left[w\left(b^{p}-a^{p}\right)^{\rho} t^{\rho}\right] \\
& \left.\times\left(t a^{p}+(1-t) b^{p}\right)^{\frac{1}{p}-1} f^{\prime}\left(t a^{p}+(1-t) b^{p}\right)^{\frac{1}{p}} d t\right] \mid \\
\leq & \frac{b^{p}-a^{p}}{2 p \mathcal{F}_{\rho, \lambda+1}^{\sigma}\left[|w|\left(b^{p}-a^{p}\right)^{\rho}\right]} \sum_{k=0}^{\infty} \frac{\sigma(k)|w|^{k}\left(b^{p}-a^{p}\right)^{\rho k}}{\Gamma(\rho k+\lambda+1)} \\
& \times \int_{0}^{1}\left[\left|(1-t)^{\rho k+\lambda}+t^{\rho k+\lambda}\right|\right]\left(t a^{p}+(1-t) b^{p}\right)^{\frac{1}{p}-1}\left(t\left|f^{\prime}(a)\right|+(1-t)\left|f^{\prime}(b)\right|\right) d t \\
\leq & \frac{b^{p}-a^{p}}{2 p \mathcal{F}_{\rho, \lambda+1}^{\sigma}\left[|w|\left(b^{p}-a^{p}\right)^{\rho}\right]} \sum_{k=0}^{\infty} \frac{\sigma(k)|w|^{k}\left(b^{p}-a^{p}\right)^{\rho k}}{\Gamma(\rho k+\lambda+1)} \\
& \times\left(\int_{0}^{1}(1-t)^{\rho k+\lambda}\left(t a^{p}+(1-t) b^{p}\right)^{\frac{1}{p}-1}\left[t\left|f^{\prime}(a)\right|+(1-t)\left|f^{\prime}(b)\right|\right] d t\right. \\
& \left.+\int_{0}^{1} t^{\rho k+\lambda}\left(t a^{p}+(1-t) b^{p}\right)^{\frac{1}{p}-1}\left[t\left|f^{\prime}(a)\right|+(1-t)\left|f^{\prime}(b)\right|\right] d t\right) \\
& =(t)
\end{aligned}
$$

So we have,

$$
\begin{aligned}
& \left|\frac{f(a)+f(b)}{2}-\frac{1}{2\left(b^{p}-a^{p}\right)^{\lambda} \mathcal{F}_{\rho, \lambda+1}^{\sigma}\left[w\left(b^{p}-a^{p}\right)^{\rho}\right]}\left[\left(J_{\rho, \lambda, a^{p}+; w}^{\sigma} f o g\right)\left(b^{p}\right)+\left(J_{\rho, \lambda, b^{p}-; w}^{\sigma} f o g\right)\left(a^{p}\right)\right]\right| \\
\leq & \frac{b^{p}-a^{p}}{2 p \mathcal{F}_{\rho, \lambda+1}^{\sigma}\left[|w|\left(b^{p}-a^{p}\right)^{\rho}\right]} \sum_{k=0}^{\infty} \frac{\sigma(k)|w|^{k}\left(b^{p}-a^{p}\right)^{\rho k}}{\Gamma(\rho k+\lambda+1)} \\
& \times\left(\int_{0}^{1}\left|t(1-t)^{\rho k+\lambda}\right| f^{\prime}(a) \mid\left(t a^{p}+(1-t) b^{p}\right)^{\frac{1}{p}-1} d t\right. \\
& +\int_{0}^{1}(1-t)^{\rho k+\lambda+1}\left|f^{\prime}(b)\right|\left(t a^{p}+(1-t) b^{p}\right)^{\frac{1}{p}-1} d t \\
& +\int_{0}^{1} t^{\rho k+\lambda+1}\left|f^{\prime}(a)\right|\left(t a^{p}+(1-t) b^{p}\right)^{\frac{1}{p}-1} d t \\
& \left.+\int_{0}^{1}(1-t) t^{\rho k+\lambda} t\left|f^{\prime}(b)\right|\left(t a^{p}+(1-t) b^{p}\right)^{\frac{1}{p}-1} d t\right)
\end{aligned}
$$


Thus,

$$
\begin{aligned}
& \left|\frac{f(a)+f(b)}{2}-\frac{1}{2\left(b^{p}-a^{p}\right)^{\lambda} \mathcal{F}_{\rho, \lambda+1}^{\sigma}\left[w\left(b^{p}-a^{p}\right)^{\rho}\right]}\left[\left(J_{\rho, \lambda, a^{p}+; w}^{\sigma} f \circ g\right)\left(b^{p}\right)+\left(J_{\rho, \lambda, b^{p}-; w}^{\sigma} f \circ g\right)\left(a^{p}\right)\right]\right| \\
\leq & \frac{b^{p}-a^{p}}{2 p \mathcal{F}_{\rho, \lambda+1}^{\sigma}\left[|w|\left(b^{p}-a^{p}\right)^{\rho}\right]} \sum_{k=0}^{\infty} \frac{\sigma(k)|w|^{k}\left(b^{p}-a^{p}\right)^{\rho k}}{\Gamma(\rho k+\lambda+1)} \\
& \times\left(b^{1-p} \beta(2, \rho k+\lambda+1)_{2} \mathcal{F}_{1}\left(1-\frac{1}{p^{\prime}}, 2 ; \rho k+\lambda+3,1-\frac{a^{p}}{b^{p}}\right)\left|f^{\prime}(a)\right|\right. \\
& +b^{1-p} \beta(1, \rho k+\lambda+2)_{2} \mathcal{F}_{1}\left(1-\frac{1}{p^{\prime}}, 1 ; \rho k+\lambda+3,1-\frac{a^{p}}{b^{p}}\right)\left|f^{\prime}(b)\right| \\
& +b^{1-p} \beta(\rho k+\lambda+2,1)_{2} \mathcal{F}_{1}\left(1-\frac{1}{p^{\prime}}, \rho k+\lambda+2 ; \rho k+\lambda+3,1-\frac{a^{p}}{b^{p}}\right)\left|f^{\prime}(a)\right| \\
& \left.+b^{1-p} \beta(\rho k+\lambda+1,2)_{2} \mathcal{F}_{1}\left(1-\frac{1}{p^{\prime}}, \rho k+\lambda+1 ; \rho k+\lambda+3,1-\frac{a^{p}}{b^{p}}\right)\left|f^{\prime}(b)\right|\right)
\end{aligned}
$$

From here,

$$
\begin{aligned}
& \left|\frac{f(a)+f(b)}{2}-\frac{1}{2\left(b^{p}-a^{p}\right)^{\lambda} \mathcal{F}_{\rho, \lambda+1}^{\sigma}\left[w\left(b^{p}-a^{p}\right)^{\rho}\right]}\left[\left(J_{\rho, \lambda, a^{p}+; w}^{\sigma} f \circ g\right)\left(b^{p}\right)+\left(J_{\rho, \lambda, b^{p}-; w}^{\sigma} f \circ g\right)\left(a^{p}\right)\right]\right| \\
& \leq \frac{b^{p}-a^{p}}{2 p \mathcal{F}_{\rho, \lambda+1}^{\sigma}\left[|w|\left(b^{p}-a^{p}\right)^{\rho}\right]} \sum_{k=0}^{\infty} \frac{\sigma(k)|w|^{k}\left(b^{p}-a^{p}\right)^{\rho k}}{\Gamma(\rho k+\lambda+1)} \\
& \times\left(b^{1-p} \frac{2}{(\rho k+\lambda+1)(\rho k+\lambda+2)}\left[{ }_{2} \mathcal{F}_{1}\left(1-\frac{1}{p^{\prime}}, 2 ; \rho k+\lambda+3,1-\frac{a^{p}}{b^{p}}\right)\right]\left|f^{\prime}(a)\right|\right. \\
& +b^{1-p} \frac{1}{\rho k+\lambda+2}\left[{ }_{2} \mathcal{F}_{1}\left(1-\frac{1}{p^{2}}, 1 ; \rho k+\lambda+3,1-\frac{a^{p}}{b^{p}}\right)\right]\left|f^{\prime}(b)\right| \\
& +b^{1-p} \frac{1}{\rho k+\lambda+1}\left[{ }_{2} \mathcal{F}_{1}\left(1-\frac{1}{p^{2}}, \rho k+\lambda+2 ; \rho k+\lambda+3,1-\frac{a^{p}}{b^{p}}\right)\right]\left|f^{\prime}(a)\right| \\
& \left.+b^{1-p} \frac{2}{(\rho k+\lambda+1)(\rho k+\lambda+2)}\left[2 \mathcal{F}_{1}\left(1-\frac{1}{p^{\prime}} \rho k+\lambda+1 ; \rho k+\lambda+3,1-\frac{a^{p}}{b^{p}}\right)\right]\left|f^{\prime}(b)\right|\right)
\end{aligned}
$$

Using (10) we obtain,

$$
\begin{aligned}
& \left|\frac{f(a)+f(b)}{2}-\frac{1}{2\left(b^{p}-a^{p}\right)^{\lambda} \mathcal{F}_{\rho, \lambda+1}^{\sigma}\left[w\left(b^{p}-a^{p}\right)^{\rho}\right]}\left[\left(J_{\rho, \lambda, a a^{p}+; w}^{\sigma} f \circ g\right)\left(b^{p}\right)+\left(J_{\rho, \lambda, b^{p}-; w}^{\sigma} f \circ g\right)\left(a^{p}\right)\right]\right| \\
\leq & \frac{b^{p}-a^{p}}{2 p \mathcal{F}_{\rho, \lambda+1}^{\sigma}\left[|w|\left(b^{p}-a^{p}\right)^{\rho}\right]} \sum_{k=0}^{\infty} \frac{|w|^{k}\left(b^{p}-a^{p}\right)^{\rho k}}{\Gamma(\rho k+\lambda+1)}\left(\sigma_{1}\left|f^{\prime}(a)\right|+\sigma_{2}\left|f^{\prime}(b)\right|+\sigma_{3}\left|f^{\prime}(a)\right|+\sigma_{4}\left|f^{\prime}(b)\right|\right)
\end{aligned}
$$

Thus, proof is completed.

Author Contributions: All authors contributed to each part of this work equally, and they read and approved the final manuscript.

Funding: This research received no external funding.

Conflicts of Interest: The authors declare no conflict of interest.

\section{References}

1. Dragomir, S.S.; Pĕarce, C.E.M. Selected Topics on Hermite-Hadamard Inequalities and Applications. Available online: https://rgmia.org/papers/monographs/Master.pdf (accessed 13 May 2019). 
2. Balarbi, S.; Dahmani, Z. On some new fractional integral inequalities. J. Inequal. Pure Appl. Math. 2009, 10, 86.

3. Set, E.;Özdemir, M.E.; Sarikaya, M.Z. Inequaities of Hermite-Hadamards type for functions whose derivatives absolute values are $m$-convex. AIP Conf. Proc. 2010, 1309, 861. [CrossRef]

4. Sarıkaya, M.Z.; Set, E.; Yaldız , H.; Basak N. Hermite-Hadamards inequalities for fractional integrals and related fractional inequalities. Math. Comput. Model. 2013, 57, 2403-2407.

5. Set, E.; Özdemir, M.E.; Dragomir, S.S. On the Hermite-Hadamard inequality and other integral inequalities involving two functions. J. Inequal. Appl. 2010, 9, 148102. [CrossRef]

6. Set, E.; Sarıkaya, M.Z.; Özdemir, M.E.; Yıldırım, H. The Hermite-Hadamard's inequality for some convex functions via fractional integrals and related result. J. Appl. Math. Stat. Inform. 2014, 10, 69-83. [CrossRef]

7. Yaldı, H.; Sarikaya, M.Z. On the Hermite-Hadamard type inequalities for fractional integral operator. J. Anal. 2018, 1-21.\%2Fs41478-018-0159-5. [CrossRef]

8. Zhang, K.S.; Wan, J.P. p-convex functions and their properties. Pure Appl. Math. 2007, 23, 130-133.

9. Işcan, I. Ostrowski type inequalities for p-convex functions. New Trends Math. Sci. 2016, 4, 140-150.

10. Işcan, I. Hermite-Hadamard type inequalities for harmonically convex functions. Hacet. J. Math. Stat. 2014, 43, 935-942.

11. Fang, Z.B.; Shi, R. On the $(p, h)$-convex function and some integral inequalities. J. Inequal. Appl. $2014,45$. [CrossRef]

12. Toplu, T. Hermite-Hadamard Type Inequality For $p$-Convex Functions via Katugampola Fractional Integrals. Available online: https:/ / www.researchgate.net/publication/322551131 (accessed on 9 May 2019).

13. Kunt, M.; Işcan, I. Hermite-Hadamard Fejér Type Inequalities for $p$-Convex Functions via Fractional Integrals. Iranian J. Sci. Tech. Trans. A-Sci. 2018, 42, 2079-2089. [CrossRef]

14. Kunt, M.; Işcan, I. Hermite-Hadamard Fejér Type Inequalities for $p$-Convex Functions via Fractional Integrals. Commun. Math. Model. Appl. 2017, 2, 1-5. [CrossRef]

15. Kunt, M.; Işcan, I. Hermite-Hadamard type inequalities for $p$-convex functions via fractional integrals. Moroccan J. Pure Appl. Anal. 2017, 3, 22-35. [CrossRef]

16. Kunt, M.; Işcan, I. Hermite-Hadamard Fejér Type Ineaqualities for p-Convex. Arab J. Math. 2017, 23, $215-230$.

17. Noor, M.A.; Noor, K.J.; Iftikhar, S. Nonconvex Functions and Integral Inequalities. Punjab Univ.J. Math. 2015, 47, 19-27.

18. Noor, M.A.; Noor, K.J.; Noor, K.I.; Mihai, M.H.; Awan, M.U. Hermite-Hadamard inequalities for differantiable $p$-convex functions using hypergeometric funtions. Available online: https://www. researchgate.net/publication/282912282 (accessed on 9 May 2019).

19. Kilbas, A.A.; Srivastava, H.M.; Trujillo, J.J. Theory and Applications of Fractional Differantial Equations; Elsevier: Amsterdam, The Netherlands, 2006.

20. Srinivasan, G.K. The gamma function: An eclectic tour. Am. Math. Mon. 2007, 114, 297-315. [CrossRef]

21. Dahmani, Z. New inequalities in fractional integrals. Int. J. Nonlinear Sci. 2010, 9, $493-497$.

22. Gorenflo, R.; Mainardi, F. Fractional Calculus: Integral and Differential Equations of Fractional Order; Springer: Wien, Austria, 1997; pp. 223-276.

23. Set, E. New inequalities of Ostrowski Type for mappings whose derivatives are s-convex in the second sense via fractional integrals. Comput. Math. Appl. 2012, 63, 1147-1154. [CrossRef]

24. Set, E.; Işcan, I.; Zehir, F. On some new inequalities of Hermite-Hadamard type involving harmonically convex functions via fractional integrals. Konuralp J. Math. 2015, 3, 42-55.

25. Raina, R.K. On generalized Wright's hypergeometric functions and fractional calculus operators. East Asian Math. J. 2005, 21, 191-203.

26. Agarwal, R.P.; Lun, M.J.; Raina, R.K. On Ostrowski type inequalities. Fasc. Math. 2016, 204, 5-27. [CrossRef]

(c) 2019 by the authors. Licensee MDPI, Basel, Switzerland. This article is an open access article distributed under the terms and conditions of the Creative Commons Attribution (CC BY) license (http:/ / creativecommons.org/licenses/by/4.0/). 